\title{
Relationship between sleep quality, sleep duration and glucose control in pregnant women with gestational diabetes
}

\author{
A. Alnaji, G.TH. Ellison, G.R. Law and E.M. Scott \\ Temporal Influences on Metabolic Events (TIME) Research Group, The Sound Asleep Laboratory, Leeds Institute of \\ Cardiovascular and Metabolic Medicine (LICAMM), University of Leeds, Leeds, LS2 9JT, UK
}

A number of studies to-date suggest that poor sleep quality and short sleep duration are associated with development of glucose intolerance and type 2 diabetes in the general population ${ }^{(1)}$. Moreover, studies in pregnant women have found associations between sleep disturbance and development of impaired glucose tolerance and gestational diabetes (GDM) ${ }^{(2)}$. However, there remains limited research on the relationship between sleep quality, sleep duration and glucose control in pregnant women with established GDM ${ }^{(3)}$. The present study therefore aimed to determine whether sleep quality and sleep duration is associated with glycemic control in pregnant women with GDM during the third trimester.

Sixty five women with GDM were recruited from Leeds Teaching Hospitals NHS Trust. Data were collected at 30-32 weeks gestation. Glucose control was assessed using 6 days of blinded continuous glucose monitoring (CGM) using an iPro2 device (Medtronic). Sleep was assessed using a validated sleep questionnaire (the Pittsburgh Sleep Quality Index, PSQI). Linear regression (adjusted for pre-pregnancy BMI and age) was performed to assess the association between total PSQI score, self-reported sleep duration and CGM measures of glucose control including: percentage of time in pregnancy target glucose range 3.5-7.8 mmol/l; area under the curve (AUC) $>7.8 \mathrm{mmol} / \mathrm{l}$; standard deviation (SD) of glucose values; average glucose level; and total number of glucose excursions.

Our results show that a higher PSQI score (indicating poorer sleep quality) was associated with: a lower proportion of time in glucose target $(\beta-0.69 ; 95 \%$ CI -1.22 to -0.15$)$; a greater AUC above target $(\beta 0.01 ; 95 \%$ CI 0.002 to 0.017$)$; a higher SD glucose signifying higher variability in glucose levels $(\beta 0.02 ; 95 \%$ CI 0.004 to 0.04$)$; a higher average glucose levels $(\beta 0.04 ; 95 \%$ CI 0.007 to 0.079 ); and a greater number of glucose excursions ( $\beta 0.46 ; 95 \%$ CI 0.066 to 0.852$)$. On the other hand longer sleep duration was associated with: a higher proportion of time within glucose target ( $\beta 1.33$; $95 \%$ CI 0.086 to 2.58 ); a smaller AUC above target $(\beta-0.02 ; 95 \% \mathrm{CI}-0.033$ to 0.002$)$; and a lower SD glucose $(\beta-0.05 ; 95 \% \mathrm{CI}-0.097$ to -0.008$)$.

\begin{tabular}{|c|c|c|c|c|c|c|}
\hline \multirow[b]{2}{*}{ Continuous glucose monitor outcome } & \multicolumn{3}{|c|}{ PSQI score } & \multicolumn{3}{|c|}{ Sleep duration } \\
\hline & $\beta$ Coef. & $95 \% \mathrm{CI}$ & P-value & $\beta$ Coef. & $95 \% \mathrm{CI}$ & P-value \\
\hline Proportion of time in glucose target & $-0 \cdot 69$ & $-1 \cdot 22$ to $-0 \cdot 15$ & $0 \cdot 012 *$ & $1 \cdot 33$ & 0.086 to 2.58 & $0.037 *$ \\
\hline AUC above target & 0.01 & 0.002 to 0.017 & $<0 \cdot 001^{*}$ & $-0 \cdot 02$ & -0.033 to 0.002 & $0 \cdot 077$ \\
\hline SD glucose & 0.02 & 0.004 to 0.04 & $0 \cdot 017^{*}$ & $-0 \cdot 05$ & -0.097 to -0.008 & $0.023^{*}$ \\
\hline Average glucose & $0 \cdot 04$ & 0.007 to 0.079 & $0 \cdot 019^{*}$ & $-0 \cdot 01$ & -0.099 to 0.072 & $0 \cdot 758$ \\
\hline Total excursions & $0 \cdot 46$ & 0.066 to 0.852 & $0 \cdot 023^{*}$ & $-0 \cdot 75$ & -1.67 to 0.174 & $0 \cdot 111$ \\
\hline
\end{tabular}

* significant P-value

This study suggests that sleep quality and sleep duration in the third trimester may be an important factor in achieving optimum glucose control in women with GDM. It offers the possibility that interventions targeted to address sleep quality/duration may help achieve tighter glucose control and thereby better pregnancy outcomes at this stage in pregnancy.

1. Reutrakul S, Van Cauter E. (2014) Interactions between sleep, circadian function, and glucose metabolism: implications for risk and severity of diabetes. Ann NY AcadSci 1311, 151-73.

2. Qiu C, Enquobahrie D, Frederick IO et al. (2010) Glucose intolerance and gestational diabetes risk in relation to sleep duration and snoring during pregnancy: a pilot study. BMC Wom Hlth 10,17.

3. Twedt R, Bradley M, Deiseroth D et al. (2015) Sleep duration and blood glucose control in women with gestational diabetes mellitus. Obstet Gynecol 126, 326-31. 